\title{
ENFOQUE DE ENFERMERÍA EN LA ATENCIÓN PRIMARIA DE DIABETES Y CORAZÓN COMO HERRAMIENTA FUNDAMENTAL PARA LA PREVENCIÓN, CUIDADO Y PROMOCIÓN
}

\section{NURSING APPROACH IN PRIMARY CARE OF DIABETES AND HEART AS A KEY TOOL FOR PREVENTION, CARE AND PROMOTION}

\author{
TITULO CORTO: ENFOQUE DE ENFERMERÍA EN LA ATENCIÓN PRIMARIA DE \\ DIABETES Y CORAZÓN
}

Daulys Alessa Mendinueta-Marin ${ }^{1}$ Zayne Alejandra Valderrama-Cadavid ${ }^{2}$, Guillermo TroutGuardiola $^{3}$, Marta Paredes-Bermúdez ${ }^{4}$

Recibido en octubre 26 de 2015

Aceptado en marzo 30 de 2016

\section{RESUMEN}

Actualmente, es evidente el impacto de la atención primaria a las personas con diabetes y la convicción de que la educación diabetológica es indispensable en su abordaje terapéutico, ya que así se puede contribuir a un plan individualizado por paciente, que permita su consecuente tratamiento y la preservación de su salud. Por esto, el profesional de enfermería y la familia deben ejercer un rol protagónico, realizando actividades preventivas, educadoras, rehabilitadoras y de atención, a través de la construcción de estrategias individuales y comunitarias. En la siguiente guía se encontrará una serie de intervenciones en atención primaria, como lo son: El papel de la enfermera y la visión de esta en la diabetes, valoración y seguimiento, aplicación del modelo de Callista Roy, proceso de atención de enfermería, recomendaciones nutricionales e indicadores sobre el paciente y la familia, con el fin de disminuir factores de riesgo y prevenir enfermedades de este tipo.

Palabras clave: Diabetes Mellitus; atención primaria; prevención; autocuidado

\section{Abstract}

Currently, it is clear the impact of primary care for people with diabetes and the belief that education about this disease is indispensable in the therapeutic approach, as this can contribute to an individualized patient plan that allows their subsequent treatment and the preservation of their health. Therefore, the nursing professional and the family should play a leading role, in performing preventive and educational activities, and rehabilitative care through building individual and community strategies. In the following guide you will find a series of interventions in primary 
care, such as: the role of the nurse and the vision of her in diabetes, assessment, monitoring and implementation of the model of Callista Roy, nursing care process, nutritional recommendations and indicators about the patient and family; in order to decrease risk factors and prevent such diseases.

Keywords: Mellitus diabetes; Primary care; Prevention; Self-care

\section{INTRODUCCIÓN}

$\mathrm{L}$ a diabetes mellitus es considerada una enfermedad crónica, que reúne una serie de alteraciones metabólicas, las cuales se caracterizan por un aumento considerable de la glucosa en sangre, es decir, esta enfermedad se produce por defectos ya sea en la secreción o acción de la insulina, por tal motivo su evolución sin un tratamiento adecuado puede conllevar a complicaciones como lo son las alteraciones cardiovasculares y renales; iniciando en lesiones ateroscleróticas que pueden ocasionar enfermedad coronaria, cerebrovascular o vascular periférica ${ }^{1,2}$.

En la diabetes mellitus el corazón es el órgano que se ve afectado con mayor frecuencia, esta enfermedad crónica es también la causante de un sinnúmero de afecciones cardiovasculares que son producto de lesiones ateromatosas ya sea en grado coronario, cerebrovascular o vascular periférico, constituyéndose como una enfermedad con gran morbilidad y que representa por su afección a otros sistemas como lo es el cardiovascular, la primera causa de mortalidad a nivel mundial, en especial en países latinoamericanos, estableciéndose como un reto el continente puesto que estas enfermedades crónicas se encuentran interrelacionadas ${ }^{3-7}$.

Según la Organización Mundial de la Salud (OMS) se calcula que en 2014 la prevalencia mundial de la diabetes fue del $9 \%$ entre los adultos mayores de 18 años; así mismo, en Colombia se estima según el Observatorio Nacional de Salud en su informe técnico que para el 2014, el padecimiento de diabetes mellitus (DM) en mujeres fue de 4,7\%, calculada a partir de la información de la revisión sistemática y de 2,0\% a partir de Registro Individual en la Prestación de Servicios; en los hombres fue de $4,3 \%$ calculada a partir de la información de la revisión sistemática y por Registro Individual en la Prestación de Servicios de 1,7\%. En cuanto al departamento del Magdalena la prevalencia para el año 2014 en mujeres fue de $1,7 \%$ y en hombres de $1,4 \% 8,9$

De acuerdo a lo anterior, el punto de partida de esta guía es la intervención a las principales causas que han permitido que la diabetes mellitus cada año afecte a más personas, entre estas se encuentra la precaria educación sobre el tema y concientización acerca de la magnitud de esta enfermedad, sus repercusiones en otros órganos o desencadenante de diversas enfermedades además del impacto en el rol familiar y comunitario.

Es por esto que es necesario orientar, educar, aconsejar y hacer partícipe al paciente a fomentar un rol de autorresponsabilidad consigo mismo, promoviendo una comunicación eficaz entre el equipo de trabajo de salud, el paciente y la familia que permitirá una continuidad en el manejo de su salud.

A partir de la prevalencia de la diabetes en la población tanto a nivel mundial, nacional y local, se planteó una búsqueda en bases de datos de revisiones sistemáticas, guías de prácticas clínicas, estudios de ensayos clínicos y artículos científicos, que permitieran observar lo siguiente:

- La atención primaria en diabetes desde el punto de vista de enfermería

- La valoración al paciente y a la familia

- Recomendaciones nutricionales y actividades de importancias promovidas por medio del proceso de atención de enfermería

- Actividad física y prevención de la Diabetes

- Implementación de indicadores y anexos de PAE (proceso de atención de enfermería) 


\section{Atención primaria desde el punto de vista de enfermería: diabetes y corazón}

La comunicación en salud es un proceso planificado y cumple un papel importante en la atención primaria, va encaminado a motivar a las personas, a adoptar nuevas actitudes, frente a la enfermedad, se desarrolla sobre las bases de las inquietudes de los individuos, el diálogo y el intercambio de salud frente al equipo interdisciplinario, pero principalmente frente el profesional de enfermería ${ }^{10}$.

El personal de enfermería tiene como funciones primordiales en la atención primaria en salud las acciones encaminadas a la promoción de la salud, la prevención de la enfermedad, la recuperación y rehabilitación de los pacientes, de tal manera que sean oportunos y accesibles todos los servicios para el individuo, la familia y la comunidad; logrando satisfacer la demanda en cada uno de los grupos etáreos ${ }^{11}$.

La diabetes afecta a todas las personas en especial las de edad avanzada lo cual es directamente proporcional al envejecimiento poblacional , por lo tanto necesita atención continua e integral, teniendo un control metabólico y dietario, puesto que la nutrición es considerada como una fuente primordial en el desarrollo y la prevención de la enfermedad cardiovascular como lo es el ejercicio para evitar y tratar las complicaciones asociadas, controlar los factores de riesgo a través de la educación terapéutica del paciente, sus familiares y cuidadores ${ }^{12-14}$.

La atención primaria se ha convertido en uno de los pilares fundamentales para la profesión de enfermería puesto que le permite al personal capacitado en esta área ser líderes en la prevención comunitaria de enfermedades crónicas, como la diabetes, y que de alguna u otra manera sin un tratamiento y seguimiento adecuado puede conllevar al padecimiento de enfermedades cardiovasculares.

En dicha enfermedad la atención por parte de enfermería representa un factor muy importante para conseguir un buen control metabólico. Por ello será necesario motivar, educar, orientar, aconsejar, participar en el manejo de la enfermedad y fomentar su auto responsabilidad y autonomía ${ }^{15}$.

En las personas con diabetes las enfermedades cardiovasculares son más frecuentes y agresivas por lo tanto las intervenciones deben ser priorizadas según el grado de riesgo del paciente, identificando el grado de conocimiento y el comportamiento para permitir una adecuada adherencia al tratamiento ${ }^{16,17}$.
De esta manera en la teoría de las necesidades básicas de Virginia Henderson brinda un apoyo fundamental a la enfermera planteando lo siguiente:

Primera valoración: En esta fase se evalúa la salud del paciente y se realiza el diagnóstico enfermero, elaborando un plan de atención que incluya las intervenciones a implementar y la educación que se brindará. Esta fase incluye todo lo relacionado a los patrones funcionales, se realiza de la siguiente manera:

Anamnesis: Se interroga al paciente, reconociendo los síntomas que ha presentado y complicaciones, antecedentes familiares, personales, hábitos, factores de riesgo, etc.

El paciente llevará en su vivienda un formato de autocontrol el cual se evaluará, además de realizar charla personalizada que incluirá el autoanálisis del paciente acerca del manejo que le ha dado a su salud y los conocimientos existentes acerca de esta.

Además, se registran medidas antropométricas, constantes vitales, se valora el cumplimiento del seguimiento de glucemias, dieta existente y tiempo de ejercicio físico diario, se realiza glucometría y valoración del pie (a medida que se hace la valoración se brinda educación y se recomienda al paciente actividades necesarias para el manejo de la diabetes). Se valorará también el cumplimiento del tratamiento farmacológico y evaluaran las zonas de punción ${ }^{18}$.

1. Diagnóstico enfermero: Se realiza con la información recolectada en el formato de patrones funcionales de la primera valoración, identificando los patrones alterados y los datos claves, posteriormente se elabora el diagnóstico enfermero y se plantean las intervenciones del plan de cuidados.

2. Intervenciones NIC (clasificación de intervenciones de enfermería): En general el plan de atención enfermera (PAE) irá dirigido a la promoción de hábitos saludables y técnicas de autocuidado, prevención de complicaciones a corto, mediano y largo plazo, control del afrontamiento y adaptación frente a la diabetes.

3. Visitas y periodicidad: Se realiza un orden de visitas de acuerdo a la condición de cada paciente, en el caso de pacientes en tratamiento con insulina se realizan de 1 a 3 días, esto dependerá del nivel de conocimiento y el estado general del paciente, pueden incrementarse las visitas o disminuir de acuerdo a el avance del paciente según 
el cumplimiento del tratamiento y las intervenciones realizadas. En el caso de pacientes con tratamiento farmacológico o con manejo de dieta y ejercicio se realizarán las visitas aproximadamente cada 7 a 15 días.

En cuanto a la segunda visita se revisa captación de la información anteriormente impartida y evalúa el nivel de comprensión, en este caso se seguirá con la educación acerca del tratamiento.

Visitas complementarias: Partiendo de la evaluación real de los resultados NOC (clasificación de resultados de enfermería), si es logrado el objetivo propuesto, el paciente se encontrará en etapa de mantenimiento y de progresión, lo cual indica que se ha alcanzado un nivel adaptativo optimo y el paciente evidencia un cumplimiento de su tratamiento.

4. Control en fase de mantenimiento y progresión: Esta etapa se encuentra fundamentada en la educación acerca de la importancia de la adherencia y cumplimiento del tratamiento, promoviendo un espíritu de autocuidado en el paciente que permita un adecuado control de la enfermedad y que disminuya la probabilidad de padecer complicaciones en órganos blanco ${ }^{19}$.

Es necesario un cambio de hábitos, para lo cual es indicado establecer estrategias que combatan las enfermedades crónicas producidas por estilos de vida no saludables, consolidándose como su principal intervención la educación en salud y la promoción del ejercicio junto con una adecuada alimentación, para esto es de gran importancia darle continuidad y vigilar las acciones de prevención reduciendo los factores de riesgo ${ }^{20,21}$.

\section{Valoración inicial a la familia}

Es necesario realizar un diagnóstico del entorno familiar del paciente aplicando el instrumento APGAR, el cual permitirá conocer el grado de funcionabilidad familiar y de esta manera identificar el impacto de la aparición de esta enfermedad crónica en uno o más de los miembros del grupo familiar.

\section{Valoración final de la familia}

En esta fase se aplicará de nuevo el instrumento APGAR familiar que nos permitirá medir la receptividad de la familia y comparar resultados desde el inicio de la valoración, el paso por cada una de las intervenciones junto con su evaluación real y el APGAR final, estableciendo de esta manera si hubo una progresión en la funcionabilidad familiar que favoreciera la salud del paciente, un estancamiento o por el contrario un retroceso en esta.

\section{Papel de enfermería en el manejo de pacientes diabéticos}

La enfermera es la líder fundamental en el equipo multidisciplinario, por lo tanto tiene una gran responsabilidad en el proceso terapéutico, puesto que a través de una relación basada en la confianza con sus pacientes puede lograr que este sea lo más receptivo posible a la educación que se le brinda, además de recolectar los datos necesarios que le permitan posteriormente durante las intervenciones ser usados generando una actitud de seguridad que contribuya al avance de la salud del paciente diabético ${ }^{22}$.

El profesional de enfermería tiene como responsabilidad generar en sus pacientes una actitud de autocuidado, propiciando un ambiente necesario que ayude aumentar su nivel en cada uno de los ámbitos (vida, salud y bienestar) en los que se desempeña a diario. Es aquí donde la atención primaria juega un papel fundamental pues permite modificar la manera en que la persona cuida de sí misma y se compromete con su salud, reconociendo que acciones aumentan o por el contrario disminuyen un nivel adecuado de salud ${ }^{23}$.

\section{La visión de enfermería en la diabetes}

Los profesionales de enfermería en atención primaria deben valorar el entorno y los recursos de la comunidad para hacer uso de un enfoque integral que beneficie a todos los pacientes diabéticos, la educación por este grupo de profesionales es clave para el control de la diabetes y sus complicaciones, lo ideal es la creación de un plan de intervenciones y de prevención que seleccione a los individuos con mayor riesgo y que incluya un refuerzo periódico a estos ${ }^{24-26}$

No solo la enfermedad afecta al individuo, sino también a su familia, por tal razón es necesario evaluar su entorno, los conocimientos que el paciente y la familia han desarrollado frente a esta situación y la manera en que es afrontada ${ }^{2}$ (Tabla 1). 
Tabla 1. Guía de atención en diabetes

\begin{tabular}{|l|}
\hline Fumador e historia del fumador \\
\hline Numero de comidas y horarios \\
\hline $\begin{array}{l}\text { Distribución de los hidratos de carbono en las } \\
\text { diferentes comidas }\end{array}$ \\
\hline Registro de ingesta diaria \\
\hline Limitaciones diabéticas \\
\hline Gustos y preferencias \\
\hline Presencia de poliuria \\
\hline Tipo de ejercicio y horario \\
\hline Hipoglucemias nocturnas \\
\hline Situaciones de fiebre \\
\hline Estado de zonas de inyección de insulina \\
\hline Exploración sistematizada de los pies \\
\hline $\begin{array}{l}\text { Tiene sentimientos de desvalorización hacia sí } \\
\text { mismo }\end{array}$ \\
\hline $\begin{array}{l}\text { Capacidad de afrontamiento/adaptación a la } \\
\text { situación actual }\end{array}$ \\
\hline Lleva hidratos de carbono de absorción rápida. \\
\hline
\end{tabular}

\section{Fases de la educación al paciente diagnosticado}

\section{En consulta:}

Educación de seguridad (individual)

- Aporta los conocimientos y desarrolla las destrezas necesarias para: Conocer a nivel básico, la diabetes, su causa y su carácter crónico (idea del paciente), Importancia de la alimentación, las necesidades individuales

- La práctica de actividad física

- Realizar correctamente las técnicas medición de glucosa en sangre

- El adecuado conocimiento del tratamiento farmacológico, si existiera y de las técnicas de inyección si se precisara

- Exploración periódica rigurosa y Cuidados de los pies

- Las primeras revisiones deberían realizarse con una periodicidad semanal y, posteriormente, se incluiría a la persona en un programa de educación estructurado grupal.

\section{Curso de Educación Terapéutica básico (grupal)}

Tiene como objetivos aumentar los conocimientos y la comprensión general sobre la DM, así como desarrollar destrezas para ejecutar las técnicas del tratamiento. Según la pirámide de aprendizaje, a las dos semanas de una intervención educativa es recordado un $2 \%$ si la actitud fue de escucha, un 10 o 20\% si han acompañado la explicación con gráficos, un $50 \%$ si se habla y un $90 \%$ si es la persona quien le enseña a otros ${ }^{27}$.

Actividades grupales específicas:

- Ejercicio físico

- Educación en nutrición

- Hábitos saludables

- Factores de riesgo

- Apoyo psicológico

Salud comunitaria: La atención primaria debe ser accesible, continúa, coordinada adecuadamente con otros aspectos de la salud, ser oportuna e integra, pues a través de esta es que se logra prevenir comunitariamente la aparición temprana o progresión de enfermedades como la diabetes ${ }^{28}$.

\section{Proceso de atención de enfermería}

El PAE está dirigido a identificar las respuestas humanas de las personas, familia y comunidad que atiende ante las posibles alteraciones de sus patrones de salud y con el propósito de realizar la intervención adecuada, a la enfermera, desde el punto de vista de su ciencia particular y su correspondiente campo de actuación ${ }^{29}$.

Dentro del proceso de atención de enfermería es necesario, con respecto al paciente diabético, realizar intervenciones en educación sanitaria que permitan una mejoría en las condiciones del mismo y en el cuidado especial de sus pies, creando además un programa de ejercicio físico que mejore la calidad de vida, mantenga un control metabólico y el peso del paciente. Estas estrategias dirigidas a la modificación de estilos de vida junto con el tratamiento farmacológico pueden prevenir o dado el caso no permitir la evolución de esta enfermedad ${ }^{30-32}$.

\section{Aplicación del modelo de Callista Roy por enfermería en pacientes con diabetes mellitus}

Este modelo permite conocer los estímulos frente a determinada situación lo cual puede ser adaptado al estado actual del paciente por medio de un plan de atención enfermero que incluya las esferas del ser. Según el modelo de Callista Roy, de acuerdo a las fases del proceso de atención de enfermería se debe realizar lo siguiente: 
Valoración: En esta fase se evaluará la parte fisiológica como primer nivel, incluye la nutrición actual del paciente, el patrón de eliminación, actividad, reposo y el nivel de protección de acuerdo a la situación socioeconómica del paciente.

En el segundo nivel se evalúan los estímulos focales es decir hace cuanto se padece de diabetes, si se han presentado complicaciones o existe falta de adherencia terapéutica. Posteriormente se evalúan los estímulos contextuales que corresponden al nivel de afrontamiento, considerando variables como sexo, edad, creencias, etc.

Finalmente se evalúan los estímulos residuales que hacen referencia a los cambios en cada actividad diaria desde que se descubrió que la persona presenta diabetes.

Diagnóstico: Deterioro adaptativo a la condición de diabético $\mathrm{R} / \mathrm{C}$ recepción inadecuada de la información en intervenciones anteriores $\mathrm{M} / \mathrm{P}$ niveles de glicemia elevados, aumento de peso, agotamiento, etc.

\section{Objetivos NOC (clasificación de los resultados de enfermería)}

- El paciente aceptará la importancia de sugerencias y la educación en salud dada en las citas de control, las aplica en su vida diaria, mantiene la dieta y cumple con el tratamiento farmacológico establecido.

- El paciente logrará niveles de glicemia capilar y en suero en los próximos controles, adaptándose a la enfermedad actual que padece.

\section{Intervenciones NIC (clasificación de las intervenciones de enfermería)}

- Elaborar guía de cuidados con términos entendibles y entregar al paciente.

- El paciente firmará un compromiso de cumplimiento de la guía de cuidados que se le entregó.

- Se realizarán visitas domiciliarias contantes para conocer el nivel de compromiso familiar y el sistema de apoyo en los cuidados al paciente.

- Ingresar al paciente a terapias de grupo con pacientes diabéticos.

- Sugerir la remisión a nutricionista, psicólogo, etc.

\section{Evaluación del plan de atención}

Esta evaluación se realizará de dos maneras, la evaluación proyectada que es elaborada al inicio de la creación del plan de atención, es decir a donde se quiere llevar al paciente; y la evaluación real que evidencia lo que de verdad se ha conseguido con las intervenciones. Con este plan se pretende que el paciente pueda:

- Comprender la importancia de la guía de cuidados entregadas en control por enfermería y observar si es evidente un progreso considerable en la salud del mismo.

- Lograr mejores niveles de glicemias y como consecuencia de hemoglobina glicosilada, adaptándose adecuadamente a su condición ${ }^{33}$.

\section{Proceso de atención a la familia}

\section{Diagnóstico}

Estará fundamentado en lo siguiente:

- La capacidad de afrontamiento de la familia frente a esta nueva enfermedad

- La capacidad de apoyo de la familia a la persona enferma

- La promoción de estilos de vida saludable desde la misma familia a cada uno de sus miembros.

- El desempeño del rol de cuidador o la promoción del autocuidado en el miembro de la familia afectado.

- La comunicación entre los miembros de la familia.

\section{Objetivos}

- La familia conocerá que es la enfermedad, cuáles son sus signos, síntomas y cuáles son los cuidados complementarios al plan individual de su familiar

- Apoyará a su miembro a través de la promoción y modificación de hábitos y actividades diarias

- Se constituirá como fuente de apoyo emocional para el paciente y afrontará de la mejor manera esta nueva etapa de la vida familiar.

\section{Intervenciones}

Las intervenciones estarán determinadas según el diagnóstico enfermero establecido y de acuerdo al tipo de familia a la que se va a enfrentar.

La familia se constituye entonces como el sistema de apoyo fundamental en el progreso positivo del paciente frente a esta enfermedad.

Por lo tanto, la principal intervención va dirigida a la educación de cada miembro de la familia quienes serán 
los encargados de formar una red complementaria y de apoyo al plan de cuidados individual del paciente.

\section{Evaluación}

- La familia logra un afrontamiento efectivo de la enfermedad de uno de sus miembros.

- La familia desarrolla una función familiar adecuada.

- La familia logra un conocimiento adecuado de la enfermedad y se constituye como una fuente de apoyo para su familiar.

\section{Recomendaciones nutricionales}

Uno de los principales cuidados de los pacientes con Diabetes Mellitus es la alimentación, para esto es necesaria una valoración que permita establecer una dieta de acuerdo a los requerimientos nutricionales del paciente y que garantice según su cumplimiento, mantener los niveles de glucosa. En este caso es recomendado que las calorías al día se deban distribuir en 4 a 5 comidas.

En el caso de la diabetes tipo 1 la dieta es más flexible y no deben modificarse gran cantidad de hábitos alimenticios, en el caso de la diabetes tipo 2 la dieta principal es hipocalórica con el fin de mantener un peso adecuado; es decir, que se reducirán aquellos alimentos ricos es grasa, carbohidratos, proteínas y se aumentaran alimentos como los vegetales, frutas y alimentos bajos en azúcar ${ }^{34}$.

\section{Actividades de importancia promovidas por el profesional de enfermería en la atención primaria de enfermedades crónicas y sus beneficios}

Como actividad complementaria a la alimentación y con múltiples beneficios a la salud, tanto para los pacientes diabéticos como para los completamente sanos, el deporte es recomendado por los profesionales de la salud puesto que previene gran cantidad de enfermedades en especial de tipo cardiovascular y le permite a la persona que lo practican mantener una calidad de vida optima y un buen estado físico ${ }^{35}$.

En el ámbito de la enfermería las diferentes teoristas han sustentado la práctica clínica y comunitaria que han permitido a los profesionales de acuerdo a los conocimientos aplicarlas y así fundamentar su labor.

Teniendo en cuenta la teoría de autocuidado de Orem ${ }^{36}$, se elabora un proceso de atención que busque explicar la capacidad de autocuidado en personas con diabetes tipo 2 y obesidad, haciendo uso de una teoría enfermera dirigida a la atención primaria, reconociendo la educación como pilar fundamental para fortalecer el conocimiento existente y comprensión sobre la diabetes. La educación es fundamental, pues primero es necesario entender y conocer la teoría, para luego ir a la práctica adecuada de actividades que le ayuden al paciente a mejorar su salud y autocuidado.

Para esto enfermería posee documentos de valoración e intervención que manejan un lenguaje que unifica el actuar enfermero, asegurando la calidad de la atención, en este caso se hace énfasis en la teoría del autocuidado, pues en el caso de las personas con diabetes el mayor déficit se encuentra en la capacidad de auto cuidarse, considerando que afecta en mayor proporción a personas mayores quienes son las que más presentan problemas de dependencia, necesitando cuidados profesionales y educación grupal favoreciendo el cambio de hábitos ${ }^{37-39}$. Además, es esencial para el paciente conocer lo importante que también se considera la exploración y cuidado de los pies de alguien con diabetes, esto se puede implementar por los profesionales de enfermería como herramienta fundamental de enseñanza en la atención primaria, llevando a cabo un seguimiento constante en pacientes con pie de riesgo y previniendo lo que se conoce como pie diabético; favoreciendo la salud y brindando una atención integral al paciente con diabetes mellitus ${ }^{40}$.

La enfermería propone abordar los problemas relacionados con la salud el ámbito comunitario previniendo en primer lugar, ejecutando su labor en diferentes escenarios, siendo autónomas, acertadas y siendo profesionales que fundamentan la práctica con conocimiento ${ }^{41}$. Estos deben reforzar los conocimientos acerca de los beneficios del autocuidado, haciendo también participes en el proceso a los familiares, con el propósito de motivar e informar acerca de la adopción e implementación de estilos de vida adecuados. Por tal motivo se deben priorizar intervenciones para implementar las guías adecuadamente y tanto los pacientes como la familia tengan una adherencia al cuidado propio y de su entorno $0^{42-44}$.

Además de la prevención, la enfermería debe desarrollar estrategias dirigidas al manejo y minimización de complicaciones, siendo la educación una herramienta primordial en el cuidado, promoción y restablecimiento de la salud. Una de las estrategias esenciales es el plan de atención con intervenciones educativas, reconociendo las necesidades individuales de cada paciente, proponiéndose alcanzar una serie de objetivos para mantener una 
calidad de vida del mismo, impidiendo el progreso de enfermedades, incentivando al empoderamiento junto con la autonomía en el paciente ${ }^{45}$, en especial en enfermedades crónicas como la diabetes y las enfermedades del corazón. Para el control de los factores de riesgo cardiovascular es necesario un cuidado global del paciente diabético, que vaya desde la adherencia terapéutica, el conocimiento de la enfermedad y otras variables. Por lo tanto, las áreas de intervención de enfermería incluyen el tratamiento farmacológico, los hábitos y estilos de vida y el apoyo social y familiar, haciendo énfasis en el autocuidado y en la autoresponsabilidad ${ }^{46,47}$.

Para lo anteriormente mencionado se debe crear un plan cuya meta sea generar conciencia en el paciente, para que este adquiera y aplique en su vida hábitos saludables, conozca la sintomatología y tratamiento de su enfermedad y sepa cómo actuar frente a esto, siendo multiplicador de una conducta de autocuidado y prevención de enfermedades en su entorno $0^{48-50}$. Las guías establecidas en la actualidad hacen énfasis en el papel importante de enfermería en la prevención primaria a través de actividades que generen en el paciente una actitud de empoderamiento dándole la información y habilidades necesarias para su autocuidado, logrando la rehabilitación de las personas ${ }^{51,52}$.

\section{Indicadores Pacientes/Familia}

\section{Pacientes:}

$\mathrm{N}^{\circ}$ De pacientes con diagnóstico principal de diabetes y que son diagnosticados con alguna enfermedad cardiovascular en un año/ $\mathrm{N}^{\circ}$ población total atendida en un año x100.

$\mathrm{N}^{\circ}$ De reingresos hospitalarios durante la atención integral de enfermería en 6 meses/ $N^{\circ}$ total de la población perteneciente al programa atención integral de enfermería x100.

$\mathrm{N}^{\circ}$ de defunciones ocurridas por diabetes en un año/ $\mathrm{N}^{\circ}$ población total en 1 año x100.

$\mathrm{N}^{\circ}$ de pacientes mayores de 16 años con diabetes ingresados al programa atención integral de enfermería en un mes $/ \mathrm{N}^{\circ}$ total de pacientes con diabetes mayores de 16 años pertenecientes al programa x 100 .

$\mathrm{N}^{\circ}$ de defunciones estimadas por $\mathrm{DM} / \mathrm{N}^{\circ}$ total de la población atendida x 100 .
$\mathrm{N}^{\circ}$ de pacientes con respuesta positiva a las intervenciones en 1 mes / $\mathrm{N}^{\circ}$ total de pacientes a los que se le iniciaron intervenciones en un mes x 100 .

$\mathrm{N}^{\circ}$ de pacientes que practican hábitos de vida saludable en 1 mes/ $\mathrm{N}$ total de pacientes a los que se les inició educación en el programa en 1 mes x100.

\section{Familia:}

$\mathrm{N}^{\circ}$ de familias de un(os) paciente(es) diabético (os) y con buena función familiar/

$\mathrm{N}^{\circ}$ total de familias pertenecientes al programa de atención integral al paciente diabético x100

$\mathrm{N}^{\circ}$ de familias de un(os) paciente(es) diabético (os) y con disfunción familiar leve/ $\mathrm{N}^{\circ}$ total de familias pertenecientes al programa de atención integral al paciente diabético x100

$\mathrm{N}^{\circ}$ de familias de un(os) paciente(es) diabético (os) y con disfunción familiar moderada/ $\mathrm{N}^{\circ}$ total de familias pertenecientes al programa de atención integral al paciente diabético x100

$\mathrm{N}^{\circ}$ de familias de un(os) paciente(es) diabético (os) y con disfunción familiar severa/ $\mathrm{N}^{\circ}$ total de familias pertenecientes al programa de atención integral al paciente diabético x100

$\mathrm{N}^{\circ}$ de familias con respuesta positiva a las intervenciones en 1 mes $/ \mathrm{N}^{\circ}$ total de familias a los que se le iniciaron intervenciones en un mes x 100

$\mathrm{N}^{\circ}$ de familias satisfechas con el programa de atención integral al paciente diabético y en proceso de adaptación en 6 meses $/ \mathrm{N}^{\circ}$ total de familias pertenecientes al programa $\mathrm{x} 100$

\section{DISCUSIÓN}

Actualmente, la evidencia científica sobre la atención primaria en diabetes es muy consistente, la mayoría de las revisiones en guías clínicas expresan de manera adecuada la importancia del actuar de la familia y el profesional de enfermería en la educación diabetológica del paciente. Así mismo, se evidencia un progreso en el cuidado donde se expresa explícitamente la importancia de contribuir a un plan individualizado que cubra tanto al paciente como a la 
familia y que permita el manejo adecuado de este, lo que anteriormente no se veía reflejado. Por lo que se reitera en cada revisión la importancia de la comunicación en salud y valorar conjuntamente todos los ámbitos externos del paciente para poder suplir todas sus necesidades. Finalmente, el papel importante en la atención primaria va encaminado a motivar a las personas y adoptar nuevas actitudes que fortalezcan la manera de enfrentar la enfermedad por parte del paciente, contribuyendo a la consecución de la promoción, prevención y recuperación de este, de forma que se tenga un alcance del individuo, la familia y la comunidad.

\section{CONCLUSIÓN}

La atención primaria se constituye como medio fundamental en la promoción de una cultura de salud en el ámbito comunitario; en este caso, la prevención de enfermedades crónicas como la diabetes y alteraciones cardiacas que se encuentran íntimamente ligadas, requieren de un compromiso de enfermería que va dirigido al liderazgo de acciones estratégicas que permitirán reducir los porcentajes establecidos epidemiológicamente, además de establecer cuidados óptimos que fortalezcan la calidad de vida del paciente.

Por lo tanto, la educación y prevención además de ser una de las actividades del equipo multidisciplinario, es en mayor medida una competencia de enfermería, puesto que está en sus manos mantener un seguimiento y apoyar a través de medidas no farmacológicas el tratamiento que lleve el paciente, pues bien se sabe que a través de la adquisición de conciencia acerca del cuidado propio de la salud y el trabajo arduo por estilos de vida saludable, se pueden lograr grandes cambios que garanticen la detección a tiempo y el no progreso de este tipo de enfermedades crónicas.

\section{DECLARACIÓN SOBRE CONFLICTOS DE INTERESES}

Los autores manifiestan no presentar ningún conflicto de interés.

\section{REFERENCIAS BIBLIOGRÁFICAS}

1. Esmatjes E, Vidal J. Patología del Corazón de Origen extracardiaco, repercusión cardiaca de la diabetes Mellitus. Revista Española de Cardiología [revista en la Internet]. 1998 [citado 2015 mayo 25]; 51(8):66170. Disponible en: http://www.revespcardiol.org/ es/patologia-del-corazon-origen-extracardiaco/ articulo/333/.

2. La Fuente N, Rafaela Cruz AG, Batres JP, Castilla ML. Guía de atención enfermera a personas con diabetes. Primera Edición. Andalucía España. Edita: Asociación Andaluza de Enfermería Comunitaria (ASANEC); 2008.

3. Zavala C, Florenzano F. Diabetes y corazón. Médica Clínica los Condes [revista en internet]. 2015 [citado 2015 noviembre 10]; 26(2): 175-185. Disponible en: http://www.sciencedirect.com/science/article/pii/ S0716864015000383?np $=\mathrm{y}$.

4. Herranz de la Morena L. Diabetes y aterosclerosis, el corazón diabético. Revista Medicina [revista en internet].2000 [citado 2015 noviembre 10]; 8(19): 10171023. Disponible en: http://www.sciencedirect.com/ science/article/pii/S0304541200701909.

5. Agudelo M, Dávila C. Carga de la mortalidad por diabetes mellitus en América Latina 2000-2011: Los casos de Argentina, Chile, Colombia y México. Gaceta Sanitaria [revista en internet]. 2015 [citado 2015 noviembre 10]; 29(3):172-177. Disponible en: http://www.sciencedirect. com/science/article/pii/S0213911115000199.

6. Piniés J, González F, Arteagoitia J. Escala de cálculo del riesgo cardiovascular para pacientes con diabetes ¿que son y de qué nos sirven?. Avances en Diabetología [revista en internet].2015 [citado 2015 noviembre 10]; 31(3): 102-112. Disponible en: http://www.sciencedirect. com/science/article/pii/S1134323015000216.

7. De la Hera J, García-Ruiz J, Delgado E. Diabetes y cribado de enfermedad coronaria: ¿dónde centramos el esfuerzo?. Española de Cardiología [revista en internet].2015 [citado 2015 noviembre 10]; 68(10): 830833. Disponible en: http://www.sciencedirect.com/ science/article/pii/S0300893215003280.

8. Centro de prensa OMS. Diabetes. Nota descriptiva $\mathrm{N}^{\circ} 312$ [Documento de internet]. 2015 [citado 2015 mayo 10]. Disponible en: http://www.who.int/mediacentre/ factsheets/fs312/es/.

9. Chaparro P, Cotes K, Vargas G, Díaz D, Cárdenas L, Escobar F, et al. Carga de enfermedad por enfermedades crónicas no transmisibles y discapacidad en Colombia. Informe técnico ONS [documento de internet]; 2015 [citado 2015 mayo 10]; 5 edición: 1-222. Disponible en: www.ins.gov.co/lineas-de-accion/.../5 to \% 20 Informe $\% 200 \mathrm{NS} \% 20 \mathrm{v}$-f1.pdf.

10. Reyes W, Abalo J, Prendes M. ¿Cómo hace efectiva la educación en salud en la atención primaria?. Cubana de Medicina General Integral [revista en la internet] .1999 [citado 2015 mayo 30]; 15(2): 176-183. Disponible en: http://www.bvs.sld.cu/revistas/mgi/vol15_2_99/ mgi10299.htm 
11. Torres M, Dandicourt C, Rodríguez A. Funciones de enfermería en la atención primaria de salud. Cubana de Medicina General Integral [revista en la internet]. 2005 [citado 2015 junio 2]; 21(3-4). Disponible en: http:// scielo.sld.cu/scielo.php?script $=$ sci_arttext\&pid $=$ S086421252005000300007\&lng = es.

12. Jansá M, Galindo M, Valverde M, Yoldi C, Vidal M, Isla P. Posicionamiento de la Sociedad Española de Diabetes (SED) sobre el perfil curricular y profesional de los profesionales de enfermería expertos en la atención de las personas con diabetes, sus familiares o cuidadores. Avances en Diabetología [revista en internet]. 2014 [citado 2015 noviembre 10]; 30(5): 150-5. Disponible en: http://www.sciencedirect.com/science/article/pii/ S1134323014000878.

13. Berciano S, Ordovás J. Nutrición y salud cardiovascular. Española de Cardiología [revista en internet].2014 [citado 2015 noviembre 10]; 67(9): 738-747. Disponible en: http://www.sciencedirect.com/science/article/pii/ S0300893214003091.

14. Gómez R, Díez J, Formiga D, Lafita J, Rodríguez L, González E, et al. Tratamiento de la diabetes tipo 2 en el paciente anciano. Medicina Clínica [revista en internet]. 2013 [citado 2015 noviembre 10]; 140 (3): 134.e1-134. e12. Disponible en: http://www.sciencedirect.com/ science/article/pii/S0025775312008524.

15. Roselló J, Alonso MA, Piquer C, Ibáñez D, García M. Atención Primaria: La Diabetes Mellitus. CS Illes Columbretes [documento en la internet]. 2015 [citado 2015 junio 3]; 1-19. Disponible en: http://files. docenciaenfermeria.webnode.es/2000010661123a13177/ DIABETES.\%20ATENCION\%20PRIMARIA.pdf.

16. López-Jaramillo P, Sánchez R, Díaz M, Cobos L, Bryce A, Parra-Carrillo J, et al. Consenso Latinoamericano de hipertensión en pacientes con diabetes tipo 2 y síndrome metabólico. Clínica e Investigación en Arteriosclerosis [revista en internet]. 2014 [citado 2015 noviembre 10]; 26(2):85-103. Disponible en: http://www.sciencedirect. com/science/article/pii/S0214916813001265.

17. Orozco D, De la Sen Fernández C, Gil V, Carratalá C, Navarro J. La diabetes mellitus y el riesgo cardiovascular. ¿Es necesario el tratamiento integral de la diabetes mellitus tipo 2 y los factores de riesgo cardiovascular? Revista Atención primaria [revista en internet].2010 [citado 2015 noviembre 10]; 42(1):16-23. Disponible en: http://www.sciencedirect.com/science/article/pii/ S0212656710700044.

18. Mata M, Caro J, Franch J, Mundet X, Santos P. Diabetes Mellitus tipo 2: Protocolo de actuación: Grupo de estudio de la diabetes en atención primaria de salud. Sociedad Catalana de medicina familiar y comunitaria [documento en la internet]. 2000 [citado 2015 junio 6]; 6-54 .Disponible en: http://www.sediabetes.org/gestor/ upload/file/00003582archivo.pdf.
19. Egea AF, Romero E. Guía básica de enfermería para personas con diabetes en atención primaria. Primera edición. Alcalá- Madrid. Colección Editorial de Publicaciones de INGESA: 1985; Edita ${ }^{\odot}$ Instituto Nacional de Gestión Sanitaria; 2009.

20. Vargas M, Adamuz C. Corazón y ejercicio. Medicine [revista en internet].2005 [citado 2015 noviembre 10]; 9(44): 2895-99. Disponible en: http://www.sciencedirect. com/science/article/pii/S0211344905738261.

21. Hernández W. Vigilancia de los factores de riesgo cardiovascular en el programa $<<$ Cartago da vida al corazón $>>$. Atención Primaria [revista en internet]. 2010 [citado 2015 noviembre 10]; 42(2): 121-122. Disponible en: http://www.sciencedirect.com/science/ article/pii/S021265670900.

22. Pérez B, García P. Grado de satisfacción de la población con los servicios de enfermería en un área de salud. Cubana de Enfermería [revista en internet]. 2005 [citado 2015 junio 7]; 21(2). Disponible en: http:// scielo.sld.cu/scielo.php?script = sci_arttext\&pid $=$ S0864$03192005000200003 \& \operatorname{lng}=$ es.

23. Cacha A, Gasco S, Hernández M, Muñoz E. Papel de enfermería en atención primaria: Plan de mejora de atención primaria en la comunidad de Madrid. Servicio Madrileño de Salud-Comunidad de Madrid [Documento en internet]. 2009 [citado 2015 junio 15]; 1-53. Disponible en: http://www.index-f.com/lascasas/ documentos/lc0434.pdf.

24. Pérez L, González-Suárez M, Duarte G, Ruymán Brito P. Prescripción de recursos comunitarios para la mejora del autocuidado en enfermedades crónicas. Gestión de un caso clínico en Atención Primaria. Enfermería Clínica [revista en internet]. 2014 [citado 2015 noviembre 10]; 24(4):254-260. Disponible en: http://www.sciencedirect. com/science/article/pii/S113086211400028X?np = y.

25. Isla P, Castella E, Insa R, López C, Icart T, Blanco M, et al. Proporción de carga asistencial generada por la diabetes mellitus en las consultas de enfermería de atención primaria de salud. Endocrinología y Nutrición [revista en internet].2007 [citado 2015 noviembre 10]; 54(1):2-9. Disponible en: http://www.sciencedirect. com/science/article/pii/S1575092207713992.

26. Costa B. Prevención de la diabetes de tipo 2. ¿Qué se puede hacer desde la atención primaria? La experiencia del DE-PLAN-CAT. Avances en Diabetología [revista en internet].2012 [citado 2015 noviembre 10]; 28(3):59-63. Disponible en: http://www.sciencedirect.com/science/ article/pii/S1134323012000671.

27. Villamil M. La enfermera de atención Primaria y la diabetes o la educación en un centro de Salud. Sociedad de enfermería familiar y comunitaria de Asturia [documento en internet]. 2014 [citado 2015 de julio 10]; 1-5. Disponible en: http://www.seapaonline. org/UserFiles/File/Dia \% 20mundial\%20diabetes \% 20 2014/D \% C3 \% ADa \% 20Mundial \% 20de \% 20la \% 20 Diabetes\%202014.doc\%20(1)pdf.pdf. 
28. Arauz A, Sanchez G, Padilla G, Fernandez M, Rosello M, Guzman S. Intervención educativa comunitaria sobre la diabetes en el ámbito de la atención primaria. Rev Panamá Salud pública [revista en internet]. 2001 [citado 2015 julio 11]; 9 (3): 145-153. Disponible en: www. scielosp.org/pdf/rpsp/v9n3/4517.pdf.

29. Amaro M. El método científico de enfermería, el Proceso de Atención, y sus implicaciones éticas y bioéticas. Cubana de Enfermería [revista en internet]. 2004 [citado 2015 junio 11]; 20(1):Disponible en:http:// scielo.sld.cu/scielo.php?script = sci_arttext\&pid = S086403192004000100010\&lng = es

30. Tizón E, Dovale MN, Fernández García M, Fernández Veiga C, López M, Mouteira M, et al. Atención de enfermería en la prevención y cuidados del pie diabético. Atención Primaria [revista en internet].2004 [citado 2015 noviembre 10]; 34(5):263-271. Disponible en: http://www.sciencedirect.com/science/article/pii/ S0212656704708458.

31. Ferrer J, Sanchez P, Pablos-Abella C, Albalat R, ElviraMacagno L, Sánchez C, et al. Beneficios de un programa ambulatorio de ejercicio físico en sujetos mayores con diabetes mellitus tipo 2. Endocrinología y nutrición [revista en internet]. 2011 [citado 2015 noviembre 10]; 58(80:387-394. Disponible en: http://www.sciencedirect. com/science/article/pii/S157509221100221X.

32. Cano R, Águila A, Miangolarra J. Efectividad de los programas de ejercicio físico en los pacientes con diabetes Mellitus. Medicina Clínica [revista en internet].2009 [citado 2015 noviembre 10]; 132(5): 188-194. Disponible en: http://www.sciencedirect.com/ science/article/pii/S0025775308000997.

33. Riffo S, Salazar A. Aplicación del proceso de enfermería según el modelo de Callista Roy en pacientes con Diabetes Mellitus. Revista Enfermería Global [revista en internet]. 2008 [citado 2015 junio 11]; (14): 1-13. Disponible en: http://revistas.um.es/eglobal/article/ viewFile/36101/34601.

34. Alfaro J, Simal A, Botella F. Tratamiento de la diabetes mellitus. Sistema nacional de Salud. Información Terapéutica del Sistema Nacional de Salud [documento en la internet] 2000 [citado 2015 junio 11]; 24(2): 3343.Disponible en: www.msssi.gob.es/biblioPublic/ publicaciones/docs/mellitus.pdf.

35. Stein AC, Molinero O, Salguero A, Corrêa MCR, Márquez S. Actividad física y salud percibida en pacientes con enfermedad coronaria. Cuadernos de Psicología del Deporte [documento en internet] .2014 [citado 2015 junio 12]; 14(1): 109-115. Disponible en: http://search. proquest.com/docview/1658735024? accountid $=43960$.

36. Compean L, Quintero L, Del Ángel B, Reséndiz E, Salazar B, González J. Educación, actividad física y obesidad en adultos con diabetes tipo 2 desde la perspectiva del autocuidado de Orem. Aquichán [revista en internet]. 2013 [citado 2015 junio 12]; 13(3): 347-362. Disponible en: http://www.scielo.org.co/scielo.php?script = sci arttext\&pid $=$ S1657-59972013000300004\&lng $=$ en .

37. Arlandis M, Sellán M, Cuevas C, Ajejas J, Gimena M, Olea J, et al. Aplicabilidad del modelo de autocuidado de D. Orem: elaboración de un registro de valoración inicial integrado para atención primaria y atención especializada. Nursing [revista en internet].2008 [citado 2015 noviembre 10]; 26(2): 54-61. Disponible en: http://www.sciencedirect.com/science/article/pii/ S0212538208706861.

38. Miguélez A, Ferrer C. La enfermera familiar y comunitaria: referente del paciente crónico en la comunidad. Enfermería Clínica [revista en internet].2014 [citado 2015 noviembre 10]; 24(1): 5-11. Disponible en: http://www.sciencedirect.com/science/article/pii/ S1130862113001915.

39. Ramón J, Fernández M, Forcada C, Pera G. Efectividad a medio plazo de una intervención educativa grupal dirigida al cuidado de los pies en pacientes con diabetes tipo 2. Enfermería clínica [revista en internet]. 2008 [citado 2015 noviembre 10]; 18(6): 302-308. Disponible en: http://www.sciencedirect.com/science/article/pii/ S1130862108758522.

40. Ledesma JL, Andujar I, Paez J. Evaluación de la exploración de los pacientes diabéticos y de la educación diabetológica en los centros del servicio Andaluz de Málaga. Internacional de ciencias podológicas [revista en internet] 2012 [citado 2015 junio 12]; 6(1): 51-57. Disponible en: https://revistas.ucm.es/index.php/RICP/ article/download/37907/36673.

41. Camargo I, Claro C. El papel autónomo de enfermería en las consultas. Avances en Enfermería [revista en internet] 2010[citado 2015 julio 22]; 28 (1): 143-150. Disponible en: http://www.scielo.org.co/pdf/aven/ v28n1/v28n1a14.pdf.

42. Garzón G, Gil A, Herrero A, Jiménez F, Cerezo M, Domínguez C. Grado de control metabólico y de factores de riesgo cardiovascular en pacientes con diabetes tipo 2 con y sin enfermedad cardiovascular. Gaceta Sanitaria [Revista en internet].2015 [citado 2015 noviembre 10]; 29(6): 425-430. Disponible en: http://www.sciencedirect.com/science/article/pii/ S0213911115001521?np=y.

43. López E, Ortiz A, López Carbajal J. Intervención educativa sobre el nivel de conocimientos en pacientes con diabetes y baja o nula escolaridad. Investigación en educación médica [revista en internet].2015 [citado 2015 noviembre 10]; 4:1-6. Disponible en: http://www.sciencedirect.com/science/article/pii/ S200750571500040X. 
44. Galindo M, Jansá M, Menéndez E. Educación terapéutica y autocuidado: resultados del estudio observacional transversal Diabetes Attitudes, Wishes and Needs 2 (DAWN2) en España. Endocrinología y nutrición [revista en internet].2015 [citado 2015 noviembre 10]; 62(8):391-399. Disponible en: http://www.sciencedirect. com/science/article/pii/S1575092215001990.

45. Achury D, Rodríguez S, Padilla M, Achury L, Leuro J, Martinez M, Et al. Efecto de un plan educativo en la capacidad de agencia de autocuidado del paciente con hipertensión arterial en una institución de segundo nivel. Aquichán [revista en internet]. 2013 [citado 2015 julio 22]; 13(3): 363-372. Disponible en: http://www. scielo.org.co/scielo.php?script $=$ sci_arttext\&pid $=$ S1657$59972013000300005 \& \operatorname{lng}=$ en .

46. Pisano M, González A. La modificación de los hábitos y la adherencia terapéutica, clave para el control de la enfermedad crónica. Enfermería Clínica [revista en internet].2014 [citado 2015 noviembre 10]; 24(1):59-66. Disponible en: http://www.sciencedirect.com/science/ article/pii/S1130862113001757.

47. Veliz-Rojas L, Mendoza-Parra S, Barriga OA. Adherencia terapéutica y control de los factores de riesgo cardiovasculares en usuarios de atención primaria. Revista Enfermería Universitaria [revista en internet].2015 [citado 2015 noviembre 10]; 12 (1):3-11. Disponible en: http://www.sciencedirect.com/science/ article/pii/S1665706315000160.
48. Pino M. Educación para la salud con personas mayores. Revista Global Healt Promotion [revista en internet] .2008 [citado 2015 julio 22]; 15(3):58-62. Disponible en: http://ped.sagepub.com/content/15/3/58.abstract.es.

49. Del Gordo-D’Amato R, Trout-Guardiola G, Acuña-Pinilla J. Eficacia de la terapia de ondas de choque como alternativa de tratamiento en lesiones del manguito rotador. Duazary. 2016 enero; 13 (1): 23-9

50. Arias-Vázquez P. Actividad física en Diabetes Mellitus tipo II, un elemento terapéutico eficaz: revisión del impacto clínico. Duazary. 2015 dic; 12 (2): 147-56

51. Rodríguez J, Grau M, Estrada J. Impacto sanitario y social de las enfermedades cardiovasculares, cuidados de enfermería. Libro enfermería clínica Avanzada.2014 [citado 2015 noviembre 10]; capitulo 4: 45-66. Disponible en: http://www.sciencedirect.com/science/article/pii/ B9788490224519000041.

52. Mata-Cases M, Artola S, Escalada J, Ezkurra P, Ferrer JC, Fornos JA, et al. Consenso sobre la detección y el manejo de la prediabetes. Grupo de Trabajo de Consensos y Guías Clínicas de la Sociedad Española de Diabetes. Revista atención primaria [revista en internet].2015 [citado 2015 noviembre 10]; 47 (7): 456-68. Disponible en: http://www.sciencedirect.com/science/article/pii/ S0212656714004016.

Para citar este artículo: Mendinueta-Marin DA, Valderrama-Cadavid ZA, Trout-Guardiola G, Paredes-Bermúdez M. Enfoque de enfermería en la atención primaria de diabetes y corazón como herramienta fundamental para la prevención, cuidado y promoción. Duazary. 2017 enero; 14 (1): 79 - 90 